Ter ahert z emi ssi on from GaAs- Al GaAs cor e- shel I nanowi res on Si (100) subst $r$ at e: Effects of appl i ed magnet i c fi el $d$ and exci t at i on wavel engt $h$

\begin{tabular}{|l|l|}
\hline 著者 & $\begin{array}{l}\text { I banes Jasher J ohn, Bal gos Na. Her mi ni a, } \\
\text { Jacul bi a Raf ael, Sal vador Ar nel, Somi nt ac } \\
\text { Ar nando, Est aci o El mer, Que Chr i st opher T. , } \\
\text { Tsuzuki Sat oshi, Yamant o Kohj i , Tani Nasahi ko }\end{array}$ \\
\hline $\begin{array}{l}\text { j our nal or } \\
\text { publ i cat i on ti tl e }\end{array}$ & Appl i ed Physi cs Let ter s \\
\hline vol une & 102 \\
\hline page r ange & $063101-1-063101-3$ \\
\hline year & $2013-02$ \\
\hline URL & ht t p: //hdl . handl e. net /10098/8074 \\
\hline
\end{tabular}




\title{
Terahertz emission from GaAs-AIGaAs core-shell nanowires on Si (100) substrate: Effects of applied magnetic field and excitation wavelength
}

\author{
Jasher John Ibanes, ${ }^{1, a)}$ Ma. Herminia Balgos, ${ }^{1}$ Rafael Jaculbia, ${ }^{1}$ Arnel Salvador, ${ }^{1}$ \\ Armando Somintac, ${ }^{1}$ Elmer Estacio, ${ }^{1}$ Christopher T. Que, ${ }^{2}$ Satoshi Tsuzuki, ${ }^{3}$ \\ Kohji Yamamoto, ${ }^{3}$ and Masahiko Tani ${ }^{3}$ \\ ${ }^{1}$ National Institute of Physics, University of the Philippines, Diliman, Quezon City 1101 Philippines \\ ${ }^{2}$ Department of Physics, De La Salle University, Manila 2401 Philippines \\ ${ }^{3}$ Research Center for Development of Far-Infrared Region, University of Fukui, Fukui 910-8507, Japan
}

(Received 26 October 2012; accepted 28 January 2013; published online 11 February 2013)

Terahertz (THz) emission from GaAs-AlGaAs core-shell nanowires (CSNW) on silicon (100) substrates was investigated using $\mathrm{THz}$ time-domain spectroscopy. The applied magnetic field polarity dependence strongly suggests that $\mathrm{THz}$ emission originated from photo-carriers in the CSNWs. Optical excitation of the GaAs-AlGaAs core-shell yielded a wider THz emission bandwidth compared with that of just the GaAs core material. This result is currently attributed to faster carrier lifetimes in the $\mathrm{AlGaAs}$ shell. The $\mathrm{THz}$ emission spectral data are supported by time-resolved photoluminescence studies. (C) 2013 American Institute of Physics. [http://dx.doi.org/10.1063/1.4791570]

Semiconductor nanowire (NW) growth and characterization continue to be the subject of fundamental and applied research. A number of growth techniques, doping methods, and heterostructure designs have been developed to attain desired crystallinity, uniformity, and electro-optic properties of these nanostructures. ${ }^{1-4}$ One such example is the coreshell NW (CSNW) design where the low band gap NW core is coated by a higher band gap shell. This results in the separation of the carriers in the core from the surface traps which enhances the recombination and transport of photo-generated carriers. ${ }^{5-7}$ Similarly, a number of characterization methods have been employed to evaluate NW morphology, crystal structure, and carrier behavior. ${ }^{8-11}$ Rather recently, opticalpump terahertz (THz)-probe measurements have been employed to survey carrier recombination and transport in NWs. ${ }^{7,12}$ However, these earlier reports have not been able to distinguish between the $\mathrm{THz}$ characteristics of the core and the shell layers. At present, even as $\mathrm{THz}$ emission has been demonstrated for NWs, ${ }^{13-15}$ that of GaAs-AlGaAs CSNWs has not been widely investigated.

In this paper, we investigate the THz emission of GaAsAlGaAs CSNWs using THz time domain spectroscopy (THzTDS). Magnetic field polarity dependence strongly suggests that the $\mathrm{THz}$ emission originates from the CSNWs and not from the two dimensional (2D) amorphous film on the substrate surface. Selective excitation of the GaAs core or the GaAs-AlGaAs core-shell allowed for the independent qualitative investigation of the core and shell carrier dynamics.

The CSNWs were grown on $\mathrm{Si}(100)$ substrates using molecular beam epitaxy via vapor-liquid-solid growth mechanism using Au nanoparticles as catalysts. A SEM micrograph of a CSNW sample is shown in Fig. 1(a). The CSNWs have diameters of $100-150 \mathrm{~nm}$, lengths of $3-8 \mu \mathrm{m}$, and a density of $1.60 \times 10^{8} \mathrm{~cm}^{-2}$. The CSNW structure consists of a GaAs core, an AlGaAs shell, and a Si-doped GaAs skin having respective nominal growth equivalent film thicknesses of $0.65 \mu \mathrm{m}, 0.84 \mu \mathrm{m}$, and $0.1 \mu \mathrm{m}$. It must be noted that these

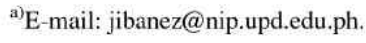

nominal growth equivalent film thicknesses would result in the formation of CSNWs with diameters of $100-150 \mathrm{~nm}$, due to the Au nanoparticles, and a 2D amorphous film on the substrate surface. The thickness of the AlGaAs shell was estimated to be $50-60 \mathrm{~nm}$ by comparing the diameters of CSNWs and uncoated GaAs NWs grown using the same growth parameters. The thickness of the $\mathrm{n}-\mathrm{GaAs}$ skin was considered thin enough to have minimal contribution to the $\mathrm{THz}$ emission. It can be observed that the CSNWs are oriented at an angle relative to the substrate surface due to preferential growth in the $\langle 111\rangle$ direction. ${ }^{16}$ The room temperature photoluminescence (PL) of the CSNW in this study is shown in Fig. 1(b). In the PL measurement, the excitation source was an $\mathrm{Ar}^{+}$laser with a wavelength of $488 \mathrm{~nm}$. PL peaks close to the corresponding nominal values of the GaAs and AlGaAs room temperature bandgaps were observed at around $800 \mathrm{~nm}$ and $870 \mathrm{~nm}$, respectively. The AlGaAs mole fraction was $\mathrm{x} \approx 0.10$ which is expected to yield a PL peak close to $800 \mathrm{~nm}$ at room temperature.

Standard THz-TDS measurements were performed in the oblique reflection excitation geometry. The excitation source was a mode-locked Ti:sapphire laser emitting $100 \mathrm{fs}$ pulses at a repetition rate of $80 \mathrm{MHz}$. The $\mathrm{THz}$ emission was detected by an optically gated LT-GaAs photoconductive dipole antenna. The $\mathrm{THz}$ induced transient photocurrent was fed to a lock-in amplifier using the $2 \mathrm{kHz}$ mechanically chopped excitation laser as reference. To study the effects of the applied $650 \mathrm{mT}$ magnetic field parallel to the substrate surface, the field polarity was switched between $B_{\text {up }}$ and $\mathrm{B}_{\text {down }}$ and compared with the $\mathrm{THz}$ emission with the no-field case (No B). The selective excitation of the GaAs core-only or the GaAs-AlGaAs core-shell was achieved by tuning the excitation wavelength to $850 \mathrm{~nm}$ or $775 \mathrm{~nm}$, respectively.

The time domain data and the corresponding Fourier transform spectra of the GaAs-AlGaAs CSNWs for $B_{\text {up }}$, $\mathrm{B}_{\text {down }}$ and No B cases are shown in Figs. 2(a) and 2(b). Similar measurements for semi-insulating (SI) GaAs substrates are also shown for comparison in Figs. 2(c) and 2(d). The THz emission from the SI-GaAs increased in intensity regardless 

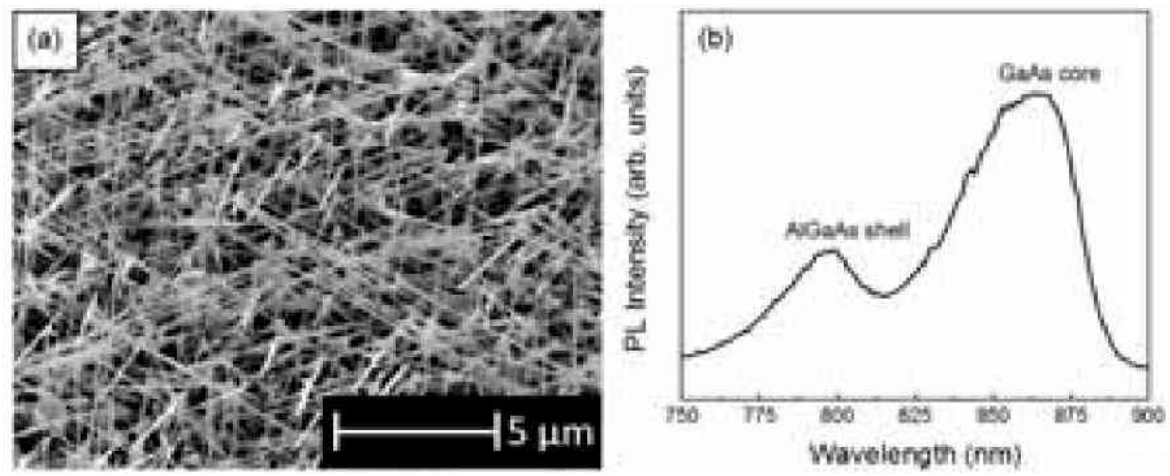

FIG. 1. (a) SEM micrograph of GaAsAlGaAs CSNWs deposited on Si (100) substrates. The CSNWS are tilted relative to substrate surface. (b) Room temperature photoluminescence spectra of the GaAs-AlGaAs CSNWs showing the peaks associated with the GaAs core and AlGaAs shell.


FIG. 2. TDS signal and THz power spectra from (a) and (b) the GaAsAlGaAs CSNWs and (c) and (d) the SI-GaAs substrate without a magnetic field (No B), with a $B_{\text {up }}$ polarity and with a $B_{\text {down }}$ polarity. The $\mathrm{THz}$ power spectra of the SI-GaAs substrate were vertically offset for ease of comparison.

of the polarity of the applied magnetic field relative to the No B case. On the other hand, for GaAs-AlGaAs CSNW, although an increase in the $\mathrm{THz}$ emission intensity was observed for the $\mathrm{B}_{\text {up }}$ polarity, the intensity decreased for the $B_{\text {down }}$ polarity; even less than the No B case.

The enhancement of $\mathrm{THz}$ emission by the application of a magnetic field on a bulk $\mathrm{THz}$ emitter has been observed and explained in a number of previous works. ${ }^{17-20}$ The anomalous magnetic field enhancement observed in this work is explained in Fig. 3. In bulk semiconductors, photo-generated carriers move perpendicular to the surface. As a result, the dipole moment of the $\mathrm{THz}$ emission is also oriented perpendicular to the surface in the absence of a magnetic field as shown in Fig. 3(a). Under magnetic field, the dipole axis becomes titled relative to the substrate surface according to the Lorentz force as shown in Figs. 3(b) and 3(c). ${ }^{17}$ However, for the CSNWs, carrier movement occurs along the CSNW's axis. Consequently, the dipole moment is also oriented along the same axis. In the case of a CSNW structure tilted relative to surface, as shown in Fig. 3(d), the dipole moment should similarly be tilted even in the absence of an applied magnetic field. If a magnetic field is then applied, the dipole moment is reoriented, as shown in Figs. 3(e) and 3(f), which result to either an increase or decrease in the detected $\mathrm{THz}$ emission depending on the field polarity. In fact, the CSNW $\mathrm{B}_{\text {down }}$ case, in Fig. 3(f), resembles the dipole moment orientation of the bulk sample for the No B case. This peculiar magnetic field polarity dependence of the $\mathrm{THz}$ emission strongly suggests that $\mathrm{THz}$ emission originates from the transient current along the NW axis and not from the 2D amorphous film on the substrate surface.

The CSNW THz emission spectra for the selective excitation of the GaAs-AlGaAs core-shell $(775 \mathrm{~nm})$ and the GaAs core-only $(850 \mathrm{~nm})$ are shown in Fig. 4(a). The THz emission from the excitation of the GaAs-AlGaAs core-shell has a wider spectrum than that from the excitation of just the core material. The higher-frequency components suggest that carriers from the AlGaAs shell material are characterized by faster carrier lifetimes (as compared to carriers from the GaAs

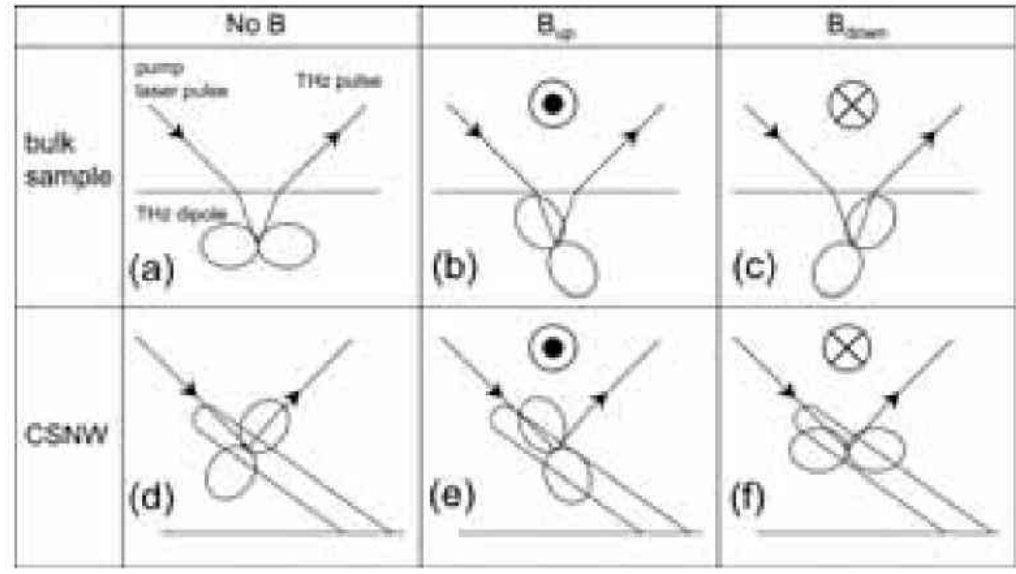

FIG. 3. The $\mathrm{THz}$ emission dipole from a planar semiconductor (a)-(c) and a CSNW (d)-(f) with and without an applied magnetic field. The dipole orientation is similar for (a) and (f); (b) and (e); and (c) and (d). 

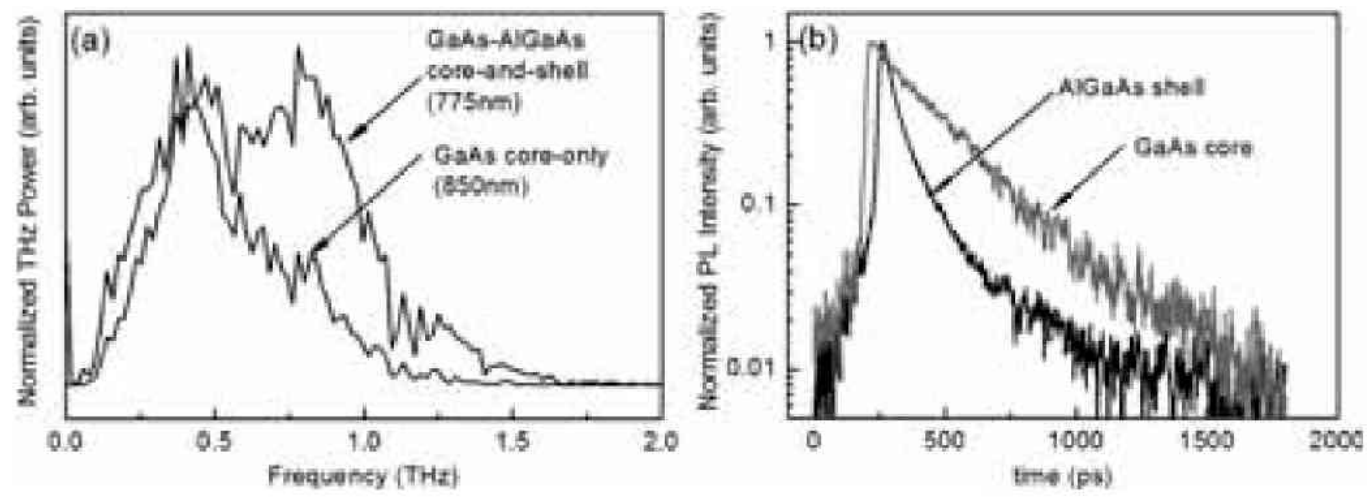

FIG. 4. (a) THz emission from the selective excitation of the GaAs-AlGaAs core-shell and of only the GaAs core. The excitation wavelengths are $775 \mathrm{~nm}$ for the GaAs-AlGaAs core-shell and $850 \mathrm{~nm}$ for only the GaAs core. (b) Time resolved PL of the GaAs core and AlGaAs shell. The PL intensity of the AlGaAs decays faster than that of the GaAs.

core). Upon ultrafast optical excitation, photo-generated carriers undergo drift transport along the nanowire axis before slowing down and eventually recombining, radiatively. In a ballistic transport picture, higher velocity carriers contribute to higher-frequency $\mathrm{THz}$ emission while slower carriers correspond to lower frequencies. As such, short carrier lifetimes should yield higher-frequency $\mathrm{THz}$ emission. The longer lifetime carriers are expected to slow down thereby producing lower frequency $\mathrm{THz}$ components. This result is supported by the time-resolved PL (TRPL) spectra of the CSNWs, shown in Fig. 4(b); where the carrier recombination lifetime in the $\mathrm{AlGaAs}$ shell is faster than that of the GaAs core. This experimental demonstration of the independent qualitative study of the carrier dynamics of the core and shell materials using $\mathrm{THz}$ emission spectroscopy may prove vital in further improvements of GaAs-AlGaAs CSNW growth designs.

An anomalous magnetic field polarity dependence of the $\mathrm{THz}$ emission intensity for CSNWs was observed. This is attributed to the $\mathrm{THz}$ emission originating from photo-carrier drift along the CSNW axis. Optical excitation of the GaAs$\mathrm{AlGaAs}$ core-shell reveal wider frequency $\mathrm{THz}$ emission, compared with the excitation of just the GaAs core. This bandwidth difference is related to the faster carrier lifetimes in the AlGaAs shell compared to the GaAs core material. The THz emission spectroscopy data are supported by TRPL measurements.

This work was undertaken with grants from the University of the Philippines (UP) Systems Grant, UP Office of the Vice-Chancellor for Research and Development, and the Department of Science and Technology.

${ }^{1}$ J. Noborisaka, J. Motohisa, S. Hara, and T. Fukui, Appl. Phys. Lett. 87, 093109 (2005).
${ }^{2}$ D. Spirkoska, J. Arbiol, A. Gustafsson, S. Conesa-Boj, F. Glas, I. Zardo, M. Heigoldt, M. H. Gass, A. L. Bleloch, S. Estrade, M. Kaniber, J. Rossler, F. Peiro, J. R. Morante, G. Abstreiter, L. Samuelson, and A. Fontcuberta i Morral, Phys. Rev. B 80, 245325 (2009).

${ }^{3}$ C. Colombo, M. Hei $\beta$, M. Graetzel, and A. Fontcuberta i Morral, Appl. Phys. Lett. 94, 173108 (2009).

${ }^{4}$ D. Lucot, F. Jabeen, J.-C. Harmand, G. Patriarche, R. Giraud, G. Faini, and D. Mailly, Appl. Phys. Lett. 98, 142114 (2011).

${ }^{5}$ P. Prete, F. Marzo, P. Paiano, N. Lovergine, G. Salviati, L. Lazzarini, and T. Sekiguchi, J. Cryst. Growth 310, 5114-5118 (2008).

${ }^{6}$ F. Jabeen, S. Rubini, V. Grillo, L. Felisari, and F. Martelli, Appl. Phys. Lett. 93, 083117 (2008).

${ }^{7}$ P. Parkinson, H. J. Joyce, Q. Gao, H. Hoe Tan, X. Zhang, J. Zou, C. Jagadish, L. M. Herz, and M. B. Johnston, Nano Lett. 9(9), 3349-3353 (2009).

${ }^{8}$ Z. Gu, P. Prete, N. Lovergine, and B. Nabet, J. Appl. Phys. 109, 064314 (2011).

${ }^{9}$ S. Perera, M. A. Fickenscher, H. E. Jackson, L. M. Smith, J. M. YarrisonRice, H. J. Joyce, Q. Gao, H. H. Tan, C. Jagadish, X. Zhang, and J. Zou, Appl. Phys. Lett. 93, 053110 (2008).

${ }^{10}$ P. Parkinson, J. Lloyd-Hughes, Q. Gao, H. Hoe Tan, C. Jagadish, M. B. Johnston, and L. M. Herz, Nano Lett. 7(7), 2162-2165 (2007).

${ }^{11}$ L. Yang, J. Motohisa, T. Fukui, L. Xi Jia, L. Zhang, M. M. Geng, P. Chen, and Y. Liang Liu, Opt. Express 17(11), 9337-9346 (2009).

${ }^{12}$ J. H. Strait, P. A. George, M. Levendorf, M. Blood-Forsythe, F. Rana, and J. Park, Nano Lett. 9(8), 2967-2972 (2009).

${ }^{13}$ G. B. Jung, Y. J. Cho, Y. Myung, H. S. Kim, Y. S. Seo, J. Park, and C. Kang, Opt. Express 18(16), 16353-16359 (2010).

${ }^{14}$ D. V. Seletskiy, M. P. Hasselbeck, J. G. Cederberg, A. Katzenmeyer, M. E. Toimil-Molares, F. Léonard, A. Alec Talin, and M. Sheik-Bahae, Phys. Rev. B 84, 115421 (2011).

${ }^{15}$ K. J. Kong, C. S. Jung, G. B. Jung, Y. J. Cho, H. S. Kim, J. Park, N. E. Yu, and C. Kang, Nanotechnology 21, 435703 (2010).

${ }^{16}$ K. Hiruma, M. Yazawa, T. Katsuyama, K. Ogawa, K. Haragachi, M. Koguchi, and H. Kakibayashi, J. Appl. Phys. 77(2), 447 (1995).

${ }^{17}$ M. B. Johnston, D. M. Whittaker, A. Corchia, A. G. Davies, and E. H. Linfield, Phys. Rev. B 65, 165301 (2002).

${ }^{18}$ M. B. Johnston, D. M. Whittaker, A. Corchia, A. G. Davies, and E. H. Linfield, J. Appl. Phys. 91(4), 2104-2106 (2002).

${ }^{19} \mathrm{C}$. Weiss, R. Wallenstein, and R. Beigang, Appl. Phys. Lett. 77(25), $4160-4162$ (2000).

${ }^{20}$ N. Sarukura, H. Ohtake, S. Izumida, and Z. Liu, J. Appl. Phys. 84, 654 (1998). 